\title{
THE LOCAL LOW-MASS BINARY PULSAR POPULATION
}

\author{
D.R. LORIMER \\ The University of Manchester, \\ Nuffield Radio Astronomy Laboratories, \\ Jodrell Bank, Macclesfield, \\ Cheshire, SK11 9DL, U.K.
}

\begin{abstract}
Using a fully self-consistent approach to account for known survey selection effects, we constrain the number and scale height of lowmass binary pulsars (LMBPs) in the local solar neighbourhood. Our results show that the local surface density of LMBPs with luminosities above $2.5 \mathrm{mJy} \mathrm{kpc}^{2}$ is $\sim 20 \mathrm{kpc}^{-2}$. Assuming that these are long-lived $\left(\lesssim 10^{10} \mathrm{yr}\right)$ objects, their local birth rate is at least $210^{-9} \mathrm{kpc}^{-2} \mathrm{yr}^{-1}$. Whilst this is in excellent agreement with the birth rate of their proposed progenitors, the low-mass X-ray binaries, there are several uncertainties involved which could significantly increase our derived birth rate, perhaps by an order of magnitude. Models in which the scale height of LMBPs above the galactic plane exceeds $500 \mathrm{pc}$ are found to be most consistent with the data. The mean space velocity at birth required to produce scale heights of this order in $10^{10} \mathrm{yr}$ is found to be $\gtrsim 80 \mathrm{~km} \mathrm{~s}^{-1}$.
\end{abstract}

\section{Introduction}

The discovery of binary and millisecond pulsars with distinctly different properties (periods typically $P \lesssim 30 \mathrm{~ms}$ and inferred magnetic field strengths $10^{8} \mathrm{G} \lesssim B \lesssim 10^{10} \mathrm{G}$ ) compared to the normal pulsars poses fundamental questions as to their origin, evolution and galactic population. In the standard binary pulsar formation model, the neutron star is formed during the supernova explosion of the initially more massive star in the binary system and remains bound to its companion. During the first $10^{7-8} \mathrm{yr}$ or so, it may be active as a normal radio pulsar spinning down to periods $\gtrsim$ few s. If the companion star is sufficiently massive to evolve into a giant and 
overflow its Roche lobe, the now old spun down neutron star can gain a new lease of life as a pulsar by spinning up as it accretes mass from the companion (Alpar et al. 1982). This is often referred to as the "recycling" scenario (Radhakrishnan 1982). Such a spin up process is already known to be happening in the $\mathrm{X}$-ray binaries (see for example Rappaport \& Joss 1983) making these systems attractive progenitors for the recycled pulsars. Within this framework, the double neutron star binaries like PSR B1913+16 evolve from the massive X-ray binaries (Smarr \& Blandford 1976); in contrast, the observationally more numerous millisecond and low-mass binary pulsars (LMBPs) evolve from the low-mass X-ray binaries (LMXBs). In the latter case, the companions are believed to be low-mass $\left(\lesssim 0.5 \mathrm{M}_{\odot}\right)$ white-dwarf stars. In this paper, we shall restrict our attention to LMBP statistics; the interested reader should read the review by Bhattacharya (these proceedings) for more details on the formation scenarios.

Despite the general acceptance of the recycling model amongst the pulsar community, one basic link that can (in principle) be tested by pulsar statistics remains poorly established: equality of LMXB-LMBP birth rates. This well known "birth rate problem" was first pointed out by Kulkarni \& Narayan (1988), who found that the LMBP birth rate appears to be at least an order of magnitude larger than the LMXBs. The difficulty in accepting this result is small-number statistics (Kulkarni \& Narayan's analysis was based on only four LMBPs and was virtually dominated by one millisecond pulsar, B1855+09). The effects of small-number statistics were well demonstrated by Johnston \& Bailes (1991), who repeated the analysis with the benefit of a revised distance scale and the completion of two high-frequency searches along the galactic plane. The effect of the new distance scale was to increase the inferred distance and hence our perceived luminosity for PSR B1855+09. Since more luminous pulsars are potentially visible out to larger distances compared with faint ones, the overall effect of B1855+09 on the LMBP birth rate was significantly reduced.

Even in the light of small-number statistics, the birth rate problem has often been advocated as evidence for alternative LMBP formation scenarios such as the accretion induced collapse of a white dwarf (Michel 1987; Grindlay \& Bailyn 1988). The recent spate of millisecond pulsar discoveries (see the review by Lyne, these proceedings) means that we now have the prospect of a more reliable evaluation of the underlying population which is the aim of this paper.

\section{Model Calculations}

There are a large number of selection effects at play in the observed pulsar sample. Most importantly, intrinsically faint pulsars cannot be detected 
out to very large distances compared to their more luminous counterparts due to the inverse square law and are therefore under-represented in a flux-limited sample. In addition, there are several more subtle deleterious effects on pulsar searches which cause deviations from a simple flux limit: dispersion smearing, sky background noise, interstellar scattering etc. (see Narayan 1987 for further details). In this paper, we are interested in the size and underlying distribution of the local LMBP population and require a correction or "scale factor" to account for those pulsars missed by the surveys as a result of these selection effects.

The method we shall adopt was originally used in an analysis of the normal pulsar population (Phinney \& Blandford 1981; Vivekanand \& Narayan 1981 ) and involves computing the volume $V$ in which a pulsar is potentially observable by the present surveys and comparing this with the effective volume $V_{\max }$ of the Galaxy. The ratio $V_{\max } / V$ serves as a good statistical estimator for the number of "similar" pulsars that exist in the volume $V_{\max }$ whose beams intersect our line of sight and we refer to this as the pulsar's scale factor $\xi$. In practice, we calculate the scale factors numerically, using a detailed computer model of the galactic pulsar population and the major surveys. A full account of the model is given in an earlier paper (Lorimer et al. 1993)

It is interesting to note that the size of the observed sample of LMBPs is approaching that used for the pioneering analysis of the normal pulsars by Gunn \& Ostriker (1970) almost 25 years ago. Given the large amount of controversy still inherent in studies of normal-pulsar statistics, we restrict the calculations in this paper to the local solar neighbourhood which is defined by a circle of radius $3 \mathrm{kpc}$ projected on the galactic plane with the Sun at the centre. Since the present all-sky surveys are most sensitive to detecting millisecond pulsars in this region, this has the effect of minimizing any systematic errors present in our calculations. The relevant parameters for the sample of 16 LMBPs that satisfy this criterion are summarised in Table 1.

\section{The Local Distribution and Number of LMBPs}

The underlying distribution of pulsars that we seed our model galaxy with is initially unknown, forcing us to make an initial guess at the distribution. However, because our scale factor calculation involves recording the parameters of the synthetic pulsars that were theoretically detectable, we can make an important consistency check as to the validity of the assumed distributions by comparing the model and true observed pulsars. For simplicity, we have opted for a uniform planar distribution together with an exponential function to model the decrease in pulsar density with increas- 
TABLE 1. Assumed parameters for the 16 LMBPs.

\begin{tabular}{lrrrrrr}
\hline \multicolumn{1}{c}{ PSR } & $\begin{array}{c}P \\
(\mathrm{~ms})\end{array}$ & $\begin{array}{c}|z| \\
(\mathrm{pc})\end{array}$ & $\begin{array}{c}L_{436} \\
\left(\mathrm{mJy} \mathrm{kpc}^{2}\right)\end{array}$ & $\begin{array}{r}\alpha / P \\
(\%)\end{array}$ & $\xi_{500}$ \\
\hline $\mathrm{J} 0034-0534$ & 1.88 & 910 & 16 & -3.0 & 64 & 90 \\
$\mathrm{~J} 0437-4715$ & 5.76 & 95 & 11 & -1.5 & 43 & 50 \\
$\mathrm{~J} 0613-0200$ & 3.06 & 350 & 100 & -1.6 & 55 & 8 \\
$\mathrm{~J} 0751+18$ & 3.47 & 720 & 40 & -2.0 & 30 & 12 \\
$\mathrm{~B} 0820+02$ & 864.87 & 520 & 62 & -2.4 & 4 & 5 \\
$\mathrm{~B} 1257+12$ & 6.22 & 605 & 7.8 & -2.4 & 23 & 40 \\
$\mathrm{~J} 1455-3330$ & 7.99 & 280 & 6.4 & -3.0 & 32 & 70 \\
$\mathrm{~J} 1713+0747$ & 4.57 & 380 & 27 & -2.0 & 9 & 10 \\
$\mathrm{~J} 1730-2304$ & 8.12 & 55 & 11 & -1.8 & 25 & 20 \\
$\mathrm{~B} 1831-00$ & 520.95 & 170 & 105 & -1.5 & 4 & 5 \\
$\mathrm{~B} 1855+09$ & 5.36 & 55 & 15 & -1.6 & 13 & 15 \\
$\mathrm{~B} 1957+20$ & 1.61 & 125 & 47 & -3.4 & 4 & 20 \\
$\mathrm{~J} 2019+2425$ & 3.93 & 105 & 17 & -1.9 & 20 & 20 \\
$\mathrm{~J} 2145-0750$ & 16.05 & 335 & 13 & -1.3 & 30 & 18 \\
$\mathrm{~J} 2317+1439$ & 3.44 & 1300 & 80 & -2.0 & 20 & 7 \\
$\mathrm{~J} 2322+2057$ & 4.81 & 475 & 2.5 & -2.0 & 12 & 130 \\
\hline
\end{tabular}

ing height $z$ above the plane. On comparing the model and observed data, we found that the precise form of planar distribution is unimportant to our results (as one would expect for a local population in a steady state); the exponential scale height $h_{z}$ of pulsars above the galactic plane, however, significantly affects matters. This is well demonstrated in Fig. 1 which shows the variation in Kolmogorov-Smirnov (KS) probability between the model and observed $z$ distributions as a function of scale height $h_{z}$.

From Fig. 1, we infer that the most favourable scale height of LMBPs is $\gtrsim 500 \mathrm{pc}$. Because of the difficulty of detecting pulsars at large $z$ heights and the uncertainty in the distance scale beyond $z \sim 1 \mathrm{kpc}$ (Taylor \& Cordes 1993), we cannot rule out the possibility of scale heights up to and beyond $\sim 1 \mathrm{kpc}$. Therefore, strictly speaking, this result should be regarded as a lower limit.

Assuming for now that $h_{z} \sim 500 \mathrm{pc}$, in Table 1 we give the scale factor of each LMBP. Summing all these scale factors, leads to an estimate for the local surface density $\Sigma \sim 20$ LMBPs kpc ${ }^{-2}$. Assuming that LMBPs are long-lived objects with lifetimes $\sim 10^{10} \mathrm{yr}$ (Camilo, Thorsett \& Kulkarni 1994), we infer a local birth rate of $210^{-9} \mathrm{LMBPs} \mathrm{kpc}^{-2} \mathrm{yr}^{-1}$, entirely consistent with the local LMXB birth rate of $410^{-9} \mathrm{LMXBs} \mathrm{kpc}^{-2} \mathrm{yr}^{-1}$ (Coté \& Pylyser 1989). Taking the uncertainty in $h_{z}$ into consideration, 


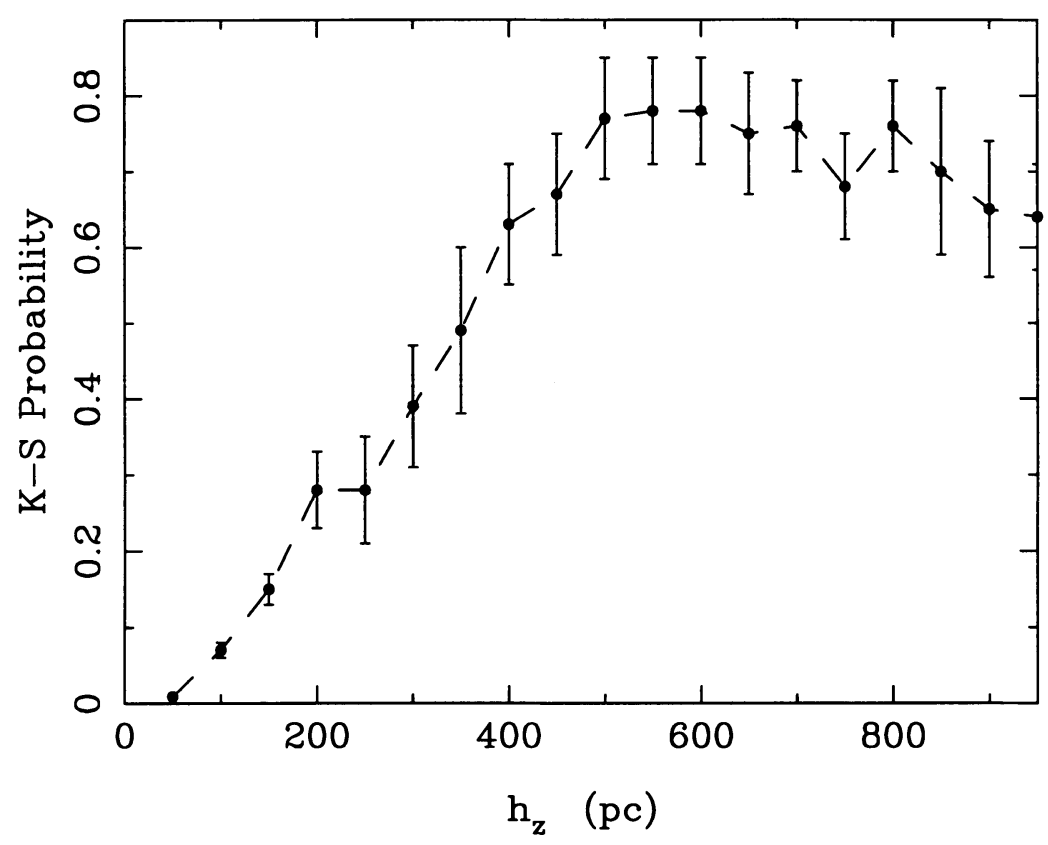

Figure 1. Results of Kolmogorov-Smirnov tests of galactic models of LMBPs with the observed $z$ distribution, as a function of the assumed scale height $h_{z}$.

we have found that the inferred local surface density from the scale factor calculations follows a simple linear relationship with $h_{z}: \Sigma \simeq\left(h_{z} / 75 \mathrm{pc}\right)+13$ LMBPs kpc ${ }^{-2}$.

Whilst our results give strong support for the LMXB-LMBP connection and the standard formation model, there are a number of caveats to be aware of. As discussed previously (Lorimer 1994) the random errors in the method are large (up to a factor of 3), due primarily to distance errors which affect the inferred luminosity and hence scale factor of each pulsar. Systematic errors can occur in several ways. Firstly, the calculation does not take into account the number of pulsars with luminosities below the minimum value $L_{\min }$ in the observed sample. In the present sample $L_{\min }=2.5 \mathrm{mJy} \mathrm{kpc}^{2}$ set by PSR J2322 +2057 . The number of pulsars with $L<L_{\min }$ is uncertain, but could be comparable to the number with $L>L_{\min }$. Secondly, we have made the simplifying assumption that all LMBPs beam to the entire sky. Given the large amount of scatter between different beaming models (see Lorimer et al. 1993) this conclusion should be regarded as provisional. A "worst case" scenario could be a beaming factor of 5. Finally, as mentioned above, there is the possibility that the scale height of LMBPs is $\gtrsim 1 \mathrm{kpc}$. From our relationship between the number of LMBPs and $h_{z}$, this could increase the scale factors by $30 \%$. All 
these systematics serve to increase the number and birth rate of LMBPs by quite uncertain factors and it is not impossible that the real LMBP birth rate could be more than an order of magnitude larger than calculated here, making it difficult to see how all LMBPs can form from LMXBs.

\section{The Birth Velocity of LMBPs}

From our analysis of the present sample, we have demonstrated that the scale height of LMBPs above the galactic plane is $\gtrsim 500 \mathrm{pc}$. If we make the assumption that the LMBP were born close to the galactic plane, at a $z$ distance of, say, $\lesssim 100 \mathrm{pc}$, they must have received a substantial kick velocity at birth in order to attain the present scale height. To estimate the size of the kick velocity, we performed Monte Carlo simulations in which 1000 LMBPs were assigned a one-dimensional (1D) kick velocity away from the galactic plane from a Gaussian distribution and allowed to oscillate in a realistic model of the galactic gravitational potential for up to $10^{10} \mathrm{yr}$. The $z$ distribution of the steady-state population was then fitted to an exponential whose scale height was compared to $h_{z}=500 \mathrm{pc}$. Since the true scale height of LMBPs is almost certainly greater than or equal to $500 \mathrm{pc}$, this allows us to set a lower limit on their birth velocities.

From the results of these simulations we find that, for an initial scale height of $100 \mathrm{pc}$ together with a Gaussian 1D velocity kick with an rms of $50 \mathrm{~km} \mathrm{~s}^{-1}$, the scale height produced is in excellent agreement with $h_{z}=500 \mathrm{pc}$. Simulations with lower initial kick velocities produce too many LMBPs at low $z$ heights. Assuming, for simplicity, that the velocity distribution is Maxwellian, the required 3D mean birth velocity is $\gtrsim \sqrt{8 / \pi} \times 50=80 \mathrm{~km} \mathrm{~s}^{-1}$. This is relatively low when compared to normal pulsars, for which $V_{\text {birth }} \sim 450 \mathrm{~km} \mathrm{~s}^{-1}$ (Lyne \& Lorimer 1994), but consistent with expectations that LMBPs occupy the low end of the velocity spectrum. Much larger velocities become less likely since they decrease the binary survival probability, reducing the number observed. Our results do not rule out the possibility that LMBPs form via AIC in which kick velocities of $\sim 100 \mathrm{~km} \mathrm{~s}^{-1}$ are entirely plausible (Woosley, Timmes \& Baron 1992). Indeed, given the uncertainties in the LMBP birth rates, we should not discount the idea that a substantial fraction of LMBPs are formed in this way.

Acknowledgements. I thank Matthew Bailes for many useful discussions and Jan van Paradijs for pointing out the possibilities of substantial kick velocities via AIC. 


\section{References}

Alpar, M.A. et al. 1982, Nat 300, 728

Camilo, F., Thorsett, S.E. \& Kulkarni, S.R., 1994, ApJ 421, L15

Coté, J. \& Pylyser, E.H.P., 1989, A\&A 218, 131

Grindlay, J.E. \& Bailyn, C.D. 1988, Nat 336, 48

Gunn, J.E. \& Ostriker, J.P. 1970, ApJ 160, 979

Johnston, S. \& Bailes, M. 1991, MNRAS 252, 277

Kulkarni, S.R. \& Narayan, R. 1988, ApJ 335, 755

Lorimer, D.R. 1994, in Lives of the Neutron Stars, M.A.Alpar, Ü. Kizlloğlu \& J. van Paradijs (Eds.), Kluwer Academic Publishers, (in press)

Lorimer, D.R. et al. 1993, MNRAS 263, 403

Lyne, A.G. \& Lorimer, D.R. 1994, Nat 369, 127

Michel, F.C., 1987, Nat 329, 310

Narayan, R. 1987, ApJ 319, 162

Phinney, E.S. \& Blandford, R.D. 1981, MNRAS 194, 137

Radhakrishnan, V. 1982, Contemp. Phys. 23, 207

Rappaport, S. \& Joss, P.C. 1983, in Accretion-Driven Stellar X-Ray Sources, W.H.G. Lewin \& E.P.J. van den Heuvel (Eds.), Cambridge University Press, p. 1

Smarr, L.L. \& Blandford, R. 1976, ApJ 207, 574

Taylor, J.H. \& Cordes, J.M. 1993, ApJ 411, 674

Vivekanand, M. \& Narayan, R. 1981, J. Astrophys. Astr. 2, 315

Woosley, S.E., Timmes, F.X. \& Baron, E. 1992, in X-ray Binaries and Recycled Pulsars, E.P.J. van den Heuvel \& S.A. Rappaport (Eds.), Kluwer Academic Publishers, p. 167 